\title{
Stress Orientations Obtained from Earthquake Focal Mechanisms: What Are Appropriate Uncertainty Estimates?
}

\author{
by Jeanne L. Hardebeck and Egill Hauksson
}

\begin{abstract}
Crustal stress orientations provide important information about the mechanics of regional deformation. Numerous methods exist for inverting earthquake focal mechanisms for stress orientation, and the more widely used methods usually obtain similar results for similar data sets. However, error estimates are highly variable, complicating the interpretation of results. The southern California stress field, for example, contains much statistically significant spatial and temporal variability according to the error estimates of one method (Michael, 1984, 1987b), but very little according to those of another (Gephart and Forsyth, 1984). To resolve whether the southern California stress field is generally homogeneous or heterogeneous, we must determine which of the error estimates best reflects the true inversion uncertainty. To do this, we tested both methods on a suite of synthetic focal mechanism data sets containing random errors. The method of Gephart and Forsyth (1984) usually provides more accurate estimates of stress orientation, especially for high-quality data sets, but its confidence regions are in most cases too large. The method of Michael $(1984,1987 b)$ is more accurate for very noisy data sets and provides a more appropriate estimate of uncertainty, implying that the stress field in southern California is probably heterogeneous.
\end{abstract}

\section{Introduction}

Knowledge of the state of stress in the Earth's crust is important for understanding the mechanics of earthquakes and regional deformation. The stress state at seismogenic depths generally cannot be measured directly. However, the orientations of the principal stress axes can be constrained from the focal mechanisms of small earthquakes occurring in the region of interest.

The two most commonly used inversion methods are a grid search method called the Focal Mechanisms Stress Inversion (FMSI) method (Gephart and Forsyth, 1984; Gephart, 1990a) and an unnamed linear inversion method (Michael, 1984, 1987b), which we refer to as the Linear Stress Inversion with Bootstrapping (LSIB) method, for convenience. These two methods typically obtain similar stress orientations for similar focal mechanism data sets. However, the uncertainty estimates are often quite different, with the confidence regions given by FMSI usually being much larger than those of LSIB.

The uncertainty estimates have important implications for understanding spatial and temporal variations in the stress field. In southern California, for example, spatial variations (Hauksson, 1990; Kerkela and Stock, 1996; Hardebeck and Hauksson, 1999, 2001) and temporal changes related to major earthquakes (Michael, 1987b; Hauksson, 1994; Zhao et al., 1997; Hardebeck and Hauksson, 2001) have been observed and found to be statistically significant according to the uncertainty estimates of LSIB and other techniques that utilize bootstrap error estimation. However, when an inversion method similar to FMSI is used, very few stress variations are found to be larger than the estimated uncertainties, and the stress field in southern California can be concluded to be generally homogeneous (Abers and Gephart, 1997).

Jones (1988) and Wyss and Lu (1995) used identical data sets to study stress orientations along the San Andreas Fault in southern California, and both studies found that the stress state was different along different segments of the fault. However, these differences appear to be significant at the $95 \%$ confidence level if LSIB is used (Jones, 1988) but not if FMSI is used (Wyss and Lu, 1995).

To resolve whether or not the observed stress field heterogeneity in southern California and along the San Andreas Fault is real, we must determine which of the uncertainty estimates best reflects the precision with which stress orientations can be determined from focal mechanisms.

The two inversion methods cannot be directly compared because they are conceptually very different. For this reason, we compare the performance of the two methods on a suite of synthetic data sets. The focus is on the appropriateness of the confidence regions computed by each method. 


\section{Inversion Methods}

All methods for inverting focal mechanisms for stress orientation rest on the assumption that earthquakes slip in the direction of the resolved shear stress on the fault plane. The orientation of the fault plane is usually not considered to be information about the stress state because it may be a randomly oriented preexisting plane of weakness. The goal of an inversion is to find the stress state which minimizes the discrepancy between the resolved shear stress direction and the slip direction for all earthquakes in the data set.

Only geometrical information is used, so the isotropic and deviatoric stress magnitudes can't be estimated, and there are only four independent model parameters. Typically, three parameters are chosen to indicate the orientations of the three principal stress axes, and the fourth parameter

$$
R=\frac{\sigma_{1}-\sigma_{2}}{\sigma_{1}-\sigma_{3}}
$$

indicates the relative magnitudes of the stress axes, where $\sigma_{1}, \sigma_{2}$, and $\sigma_{3}$ are the magnitudes of the maximum, intermediate, and minimum compressive stresses, respectively. For a reliable inversion, the orientations of the fault planes must be adequately diverse to sample the entire stress tensor.

There are two basic assumptions in the inversion procedures. The first is that the four stress parameters are constant over the spatial and temporal extent of the data set. The validity of this assumption can be tested for a particular data set using the misfit of the best-fitting stress model. A high average misfit indicates that the stress field is highly heterogeneous and hence that the stress inversion result may not be meaningful. The maximum acceptable misfit has been quantified for LSIB by Michael (1991) and for FMSI by Wyss et al. (1992), Gillard et al. (1996), and Lu et al. (1997).

The second assumption-that slip is in the direction of resolved shear stress-implies isotropic fault planes, without a preferred direction of slip. Real faults may not be isotropic, but unless there is systematic anisotropy in the set of faults used in an inversion, this simply adds some random noise. Pollard et al. (1993) demonstrated that fault length-to-width ratios and proximity to the free surface can also cause discrepancies between the slip direction and the direction of resolved shear stress but that these errors are small relative to common measurement errors.

A persistent problem with focal mechanism inversions is the ambiguity between the fault and auxiliary planes. For discussion of fault plane selection methods and the impact of incorrectly chosen planes on inversion results, see the articles by Michael (1987a) and Lund and Slunga (1999).

\section{Linear Inversion Method}

The LSIB method solves for the stress tensor using a linear, least-squares inversion (Michael, 1984). The stress inversion problem is nonlinear but can be linearized by assuming that the magnitude of the shear traction on each fault plane is approximately the same. This assumption has some physical basis because the planes that fail in earthquakes are likely to be near some failure stress.

The inversion setup is:

$$
\left(\begin{array}{c}
\overline{\mathbf{A}}_{1} \\
\overline{\mathbf{A}}_{2} \\
\vdots
\end{array}\right) \overline{\boldsymbol{\Sigma}}=\left(\begin{array}{c}
\hat{\mathbf{s}}_{1} \\
\hat{\mathbf{s}}_{2} \\
\vdots
\end{array}\right)
$$

where $\overline{\overline{\mathbf{A}}}_{j}$ is a matrix determined from the orientation of the $j$ th fault plane, $\bar{\Sigma}$ is a vector of the stress tensor parameters, and $\hat{\mathbf{s}}_{j}$ is a unit vector in the slip direction of the $j$ th event. The least-squares inversion minimizes the difference between $\hat{\mathbf{s}}_{j}$ and $\overline{\boldsymbol{\tau}}_{j}=\overline{\overline{\mathbf{A}}}_{j} \overline{\boldsymbol{\Sigma}}$, the vector of resolved shear stress on the $j$ th plane.

Confidence regions are determined using a bootstrap technique (Michael, 1987b). The data set is resampled, with replacement, hundreds or thousands of times to simulate repeated samples of the population from which the data came, and each resampling is inverted for the stress tensor. The $X \%$ of inversion results closest to the initial result, where closeness is defined by the normalized tensor dot product for stress tensor representations with zero isostatic component, are used to define the $X \%$ confidence region. (The symbol $X$ is used in this article to represent the confidence level when statements are made which hold for any level of confidence.) This technique is appropriate for data containing errors because the observed variation in the data is used to estimate the confidence regions. We use 2000 bootstrap resamplings, which is adequate to produce stable confidence regions up to the 95\% level [Michael, 1987b]. To address the fault plane ambiguity of the focal mechanisms, each nodal plane has a $50 \%$ probability of being chosen during bootstrap resampling.

The assumptions of this method are that the tractions on all fault planes are of similar magnitude, and that the assumptions of bootstrap error estimation apply. This requires the data set to be adequately representative of the population and to exhibit the full range of error.

\section{Grid Search Method}

The other widely used method, FMSI, uses a grid search over stress field parameter space to find the stress tensor that minimizes the misfit between model and data (Gephart and Forsyth, 1984; Gephart, 1990a). The misfit computed for each event is the minimum angle of rotation of the focal mechanism, about any axis, necessary to bring the slip direction into alignment with the resolved shear stress on the fault plane. Fault plane ambiguity is addressed by using the nodal plane with the smaller misfit. The L1 norm is used to find the total misfit, $\Sigma$, for a given stress state.

We perform an initial search over all of parameter space using a coarse grid with $10^{\circ}$ spacing and a second finer search with $5^{\circ}$ spacing in the vicinity of the best-fit solution. 
Confidence regions are estimated from the distribution of misfit values. The statistics are based on those derived for the L1 norm by Parker and McNutt (1980). For $N$ measurements $x_{1}, x_{2}, \ldots, x_{N}$, where each measurement $x_{j}$ is a normally distributed random variable with mean 0 and standard deviation $\sigma_{j}$, the random variable

$$
m=\sum_{j=1}^{N} \frac{\left|x_{j}\right|}{\sigma_{j}}
$$

has an expected value of

$$
E[m]=\left(\frac{2}{\pi}\right)^{1 / 2} N
$$

and a variance of

$$
\operatorname{var}[m]=\sigma^{2}=\left(1-\frac{2}{\pi}\right) N .
$$

This distribution is approximately Gaussian for $N \geq 10$. If $x_{1}, x_{2}, \ldots, x_{N}$ represent the individual mechanism misfits due to data errors, equations (4) and (5) describe the probability distribution for the total misfit of the correct stress state.

In order to use these statistics, however, the standard deviation of the misfit due to data errors, $\sigma_{\text {est }}$ (assuming $\sigma_{j} \approx \sigma_{\text {est }}$, for all $j$ ), needs to be estimated. Assuming that the total misfit value for the best-fitting stress state, $\Sigma_{\min }$, is the expected value of the total misfit for the correct stress state, then

$$
\begin{aligned}
\Sigma_{\min }=E\left[\sum_{j=1}^{N}\left|x_{j}\right|\right]= & E\left[m \sigma_{\text {est }}\right] \\
& =\sigma_{\text {est }} E[m]=\sigma_{\text {est }}\left(\frac{2}{\pi}\right)^{1 / 2} N
\end{aligned}
$$

and therefore

$$
\sigma_{\mathrm{est}}=\frac{\Sigma_{\min }}{(2 / \pi)^{1 / 2} N}
$$

Since the model has four parameters, FMSI uses

$$
\sigma_{\mathrm{est}}=\frac{\Sigma_{\min }}{(2 / \pi)^{1 / 2}(N-4)},
$$

which makes a noticeable difference in the confidence regions only for small data sets.

The $X \%$ confidence region is defined by stress states with misfits less than $\Sigma_{X}$, where there is a $(100-X) \%$ chance that the correct stress state could have a misfit greater than $\Sigma_{X}$ due to data errors. This is equivalent to a (100-
$X) \%$ probability of obtaining $m>M_{X} \equiv \Sigma_{X} / \sigma_{\text {est }}$ from the normal distribution of $m$, so

$$
M_{X}=z_{X} \sigma+E[m]
$$

where $z_{X}$ is the number of standard deviations corresponding to $X \%$ of the area under the positive half of a normal distribution (since no misfits are less than $\Sigma_{\min }$ ), for example, $z_{95}=1.96$.

The threshold value is found by substituting equations (4) and (5) into equation (9):

$$
\frac{\Sigma_{X}}{\sigma_{\text {est }}}=z_{X}\left[\left(1-\frac{2}{\pi}\right) N\right]^{1 / 2}+\left(\frac{2}{\pi}\right)^{1 / 2} N
$$

and combining them with Equation (8)

$$
\Sigma_{X}=\left(\frac{z_{X}(\pi / 2-1)^{1 / 2} N^{1 / 2}+N}{N-4}\right) \Sigma_{\min }
$$

The assumptions that go into these confidence regions are that the individual event misfits due to data errors are normally distributed with a constant standard deviation and that the total misfit of the best-fit solution, $\Sigma_{\min }$, is the expected value of the total misfit of the correct solution, $\sigma_{\mathrm{est}} E[m]$.

\section{Testing the Inversion Methods}

We examine the accuracy of the two inversion methods and the appropriateness of their uncertainty estimates by testing them on a suite of synthetic focal mechanism data sets. The design of synthetic data is inevitably subjective, but we attempt to create synthetic data sets that resemble real noisy data as much as possible.

\section{Synthetic Data Sets}

Each synthetic data set consists of a collection of focal mechanisms all consistent with a single specified stress state. The orientation of the principal stress axes is chosen randomly from a uniform spatial distribution, and a value is selected for $R$.

For most tests, the fault plane orientations are chosen at random from a uniform spatial distribution. We make the somewhat counterintuitive choice of uniformly distributed planes based on the observation that small earthquakes, in contrast with larger events, tend to occur on diverse fault planes. It is these smaller events that are typically used as stress indicators. The choice of uniformly distributed planes is at odds with the assumption of LSIB of similar magnitude shear stress on all planes and should therefore be the toughest test of this technique.

For some tests, fault planes are selected to be more uniform. Candidate planes are selected randomly as before, and 
the normal and shear stress on each plane is found, assuming that the deviatoric stress is $\sim 0.65$ of the isostatic stress. The probability of including a plane in the data set is proportional to the ratio of the shear stress to the normal stress. This results in a data set containing a larger percentage of faults that are close to the optimal orientation for failure.

Once the fault planes have been selected, the assumed stress tensor is projected onto each fault plane. The rake of each event is chosen to be in the direction of resolved shear stress, consistent with the assumption of the stress inversions.

Random errors are introduced into the data sets by rotating each mechanism about some axis. The axes are chosen from a uniform spatial distribution. An exponential probability distribution is used to select rotation angles. Random polarity and location errors lead to errors in first-motion focal mechanisms with an exponential, rather than normal, distribution (Fig. 1). Additionally, studies using real data find that the residuals for the best-fitting stress state are often exponentially distributed (Gephart and Forsyth, 1984; Gephart, 1990b).

The fault plane or the auxiliary plane is listed in the data set, with a $50 \%$ probability of each. The inversions are performed assuming that the correct plane is not known. The LSIB method may use either plane during bootstrap resampling, and FMSI may use either plane in computing misfits.

Data sets with varying amounts of error, number of events, and values of $R$ are created to observe whether these parameters affect the inversion results. Mechanism errors of $5^{\circ}, 10^{\circ}, 15^{\circ}, 20^{\circ}, 30^{\circ}$, and $40^{\circ}$ are used. These numbers represent the parameter $\mu$, where the exponential probability distribution function is defined as $\frac{1}{\mu} \mathrm{e}^{-x / \mu}$. Values of $0,0.2$, $0.4,0.5,0.6,0.8$, and 1 are used for $R$. Data sets include 20, 50,100 , or 300 events. Fifty data sets are created for most combinations of values for these three parameters.

\section{Testing}

The accuracy of the inversion methods and the appropriateness of their uncertainty estimates are tested by inverting each of the synthetic data sets using both methods. First, we test the accuracy of the inversion results, defined as the angle between the correct and best-fitting stress orientations and the difference in $R$ value.

We then test the appropriateness of the uncertainty estimates. If the confidence regions are appropriate, the correct stress state should fall within the $X \%$ confidence region for approximately $X \%$ of the data sets, for all $X$. The number of times the correct stress state falls into the $X \%$ confidence region, plotted versus $X$, should approximate a straight line. If the confidence regions are systematically too large or too small, it will fall above or below this line, respectively, as illustrated in Figure 2.

When evaluating FMSI, the correct stress state is considered to be inside the $X \%$ confidence region if $\Sigma_{\text {cor }} \leq \Sigma_{X}$, where $\Sigma_{\text {cor }}$ is the total misfit of the nearest grid point to the

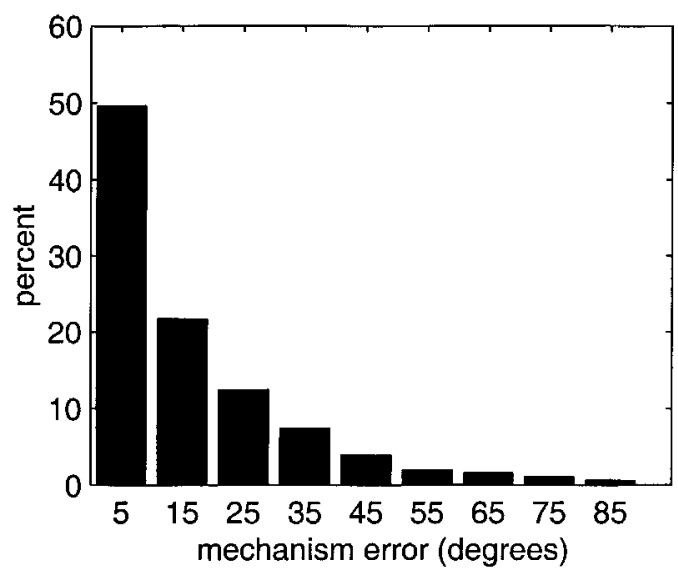

Figure 1. Histogram of the errors in first-motion focal mechanisms resulting from polarity and takeoff angle errors. Forty-one diverse focal mechanisms were chosen, and first-motion polarities for each were assigned to stations using the station distribution of Southern California Seismic Network (SCSN) stations for 41 actual southern California events. Random errors in polarity and in takeoff angle (i.e., in event location or velocity model) were added. Each first-motion observation has a $20 \%$ chance of being reversed. The error in azimuth to each station is normally distributed with a standard deviation of $2^{\circ}$, and the error in takeoff angle is normally distributed with a standard deviation of $10^{\circ}$. New focal mechanisms were determined using the FPFIT software package (Reasenberg and Oppenheimer, 1985), and the mechanism error was computed by determining the minimum rotation angle between the computed and correct mechanisms. This was repeated 100 times for the 41-event data set, and a histogram of mechanism error was compiled. Note that the mechanism errors resemble an exponential distribution, not a normal distribution.

correct stress state. For LSIB, the correct stress state is considered to be inside the $X \%$ confidence region if it is closer to the best-fit stress state than $(100-X) \%$ of the bootstrap resampling inversion results, closeness again measured by the normalized tensor dot product.

\section{Results}

\section{Accuracy}

The accuracy with which both inversion methods determine the stress parameters (Figs. 3, 4) is generally good. The accuracy of FMSI is on average slightly better, with a mean orientation error over all the test data sets of $11^{\circ}$ and a mean $R$ error of 0.09 . The LSIB method has a mean orientation error of $13^{\circ}$ and a mean $R$ error of 0.11 , and FMSI is consistently very accurate (error $\leq 5^{\circ}$ for $90 \%$ of the tests) in favorable conditions, such as large data sets $(N=300)$ or small mechanism error $\left(5^{\circ}\right)$. However, the FMSI estimates of $R$ are poor for the axisymmetric case $(R=0$ or $R=1)$. 


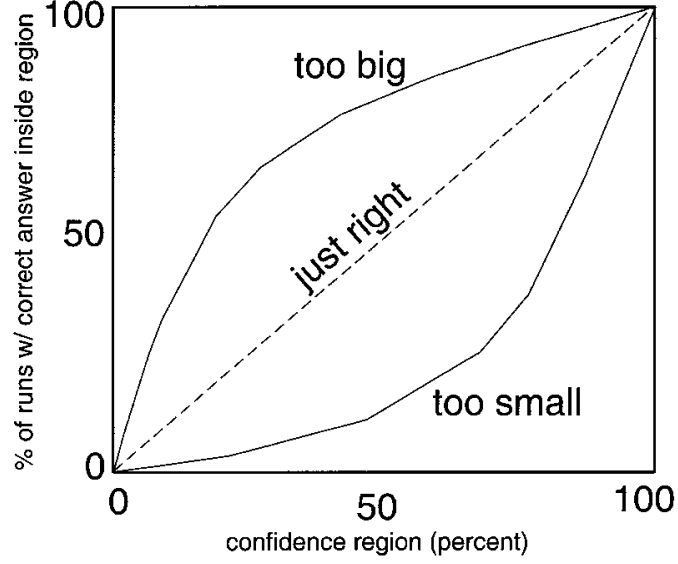

Figure 2. An illustrative plot of the number of times the correct stress state falls within a given confidence region versus the percent confidence level. If the uncertainty estimates are correct, the plot should approximate a straight line. If the plot falls above this line, this means that the correct answer falls within the $X \%$ confidence region $\gg X \%$ of the time and the confidence regions are too big. Similarly, if the plot falls below the line, the correct answer falls within the $X \%$ confidence region $\ll X \%$ of the time and the confidence regions are too small.

The accuracy of both methods improves with increasing data set size, as one might expect, with the most improvement occurring between $N=20$ and $N=50$. The accuracy deteriorates with increasing mechanism error, as also might be expected. The accuracy of the FMSI results degrade more quickly with increasing mechanism error, making LSIB more accurate for data sets with error greater than $\sim 25^{\circ}$.

The two inversion techniques make different choices about the parameter to minimize and how to handle nodal plane ambiguity, but both sets of assumptions are apparently reasonable. The linearization scheme employed by LSIB appears not to have had a major adverse effect on the accuracy of the inversion results, even though the test data sets with uniformly distributed planes should have been particularly tough on the linearization approximation.

The levels of accuracy for LSIB and FMSI indicate that the difference in confidence region size does not stem from a difference in accuracy. One might expect the more accurate method to have the smaller confidence regions, but in this case, it has the larger. This further suggests that either the confidence regions of FMSI are too large or those of LSIB are too small.

\section{Confidence Regions}

In most tests of the confidence regions (Figs. 5-8), the uncertainty estimates of LSIB are the more appropriate. The correct stress state generally falls into the $X \%$ confidence region for $(X \pm 10) \%$ of the inversions, indicating that the confidence regions of LSIB are approximately correct.

One exception is for large data sets, $N=300$, in which case the confidence regions are much too small (Fig. 5). In the tests with more uniform fault planes, the confidence regions are too small if all four stress parameters are considered, but not if the stress orientations are considered alone (Figure 8). This indicates that the parameter $R$ is not as well constrained as implied by the uncertainty estimates for data sets containing many similarly oriented events.

The appropriateness of the confidence regions of LSIB does not degrade with increasing mechanism error (Fig. 6), indicating that the bootstrap technique successfully incorporates data errors into the uncertainty estimates.

In most cases, the confidence regions of FMSI are much too large, with the correct stress state falling within the $X \%$ confidence region for $\gg X \%$ of the inversions. For example, the given $68 \%$ confidence regions often contain the correct result $90-95 \%$ of the time. In the axisymmetric case $(R=$ 0 or $R=1$ ), the FMSI confidence regions are apparently too small when all four model parameters are considered (Fig. 7). However, when only the stress orientations are considered, the confidence regions appear more appropriate. This, along with the observation that FMSI determines axisymmetric values of $R$ with poor accuracy (Fig. 4), indicates that the problem is primarily incorrect estimation of $R$.

\section{Discussion}

From the aforementioned results, it is apparent that the two inversion methods have different strengths and weaknesses. The FMSI method usually provides more accurate estimates of stress orientation, especially in favorable conditions such as large data sets and high-quality focal mechanisms. The LSIB method, although less accurate on average, provides a better estimate of uncertainty and is more accurate for data sets containing large errors. The method that is best to use for a particular study depends on the quality of the data and the relative importance of accuracy and error estimation.

An ideal inversion method would combine the strengths of both a grid search over parameter space and a nonparametric approach to error estimation. Unfortunately, nonparametric techniques such as bootstrap resampling require repeating the inversion hundreds or thousands of times, and the grid search method is very slow. As computational speed increases, combining grid search with nonparametric error estimation will become more practical. Another possibility is to speed up the search over parameter space using techniques such as genetic algorithms (Michael, 2000).

\section{Problems with LSIB}

The somewhat lower average accuracy of LSIB may be due in part to the approximation of equal shear stress on all fault planes used in the linearization of the problem. Uniformly distributed fault planes violate this approximation in the extreme, so the results of LSIB for the test data sets probably represent the worst-case accuracy. The handling of the fault plane ambiguity problem by the LSIB method, which 

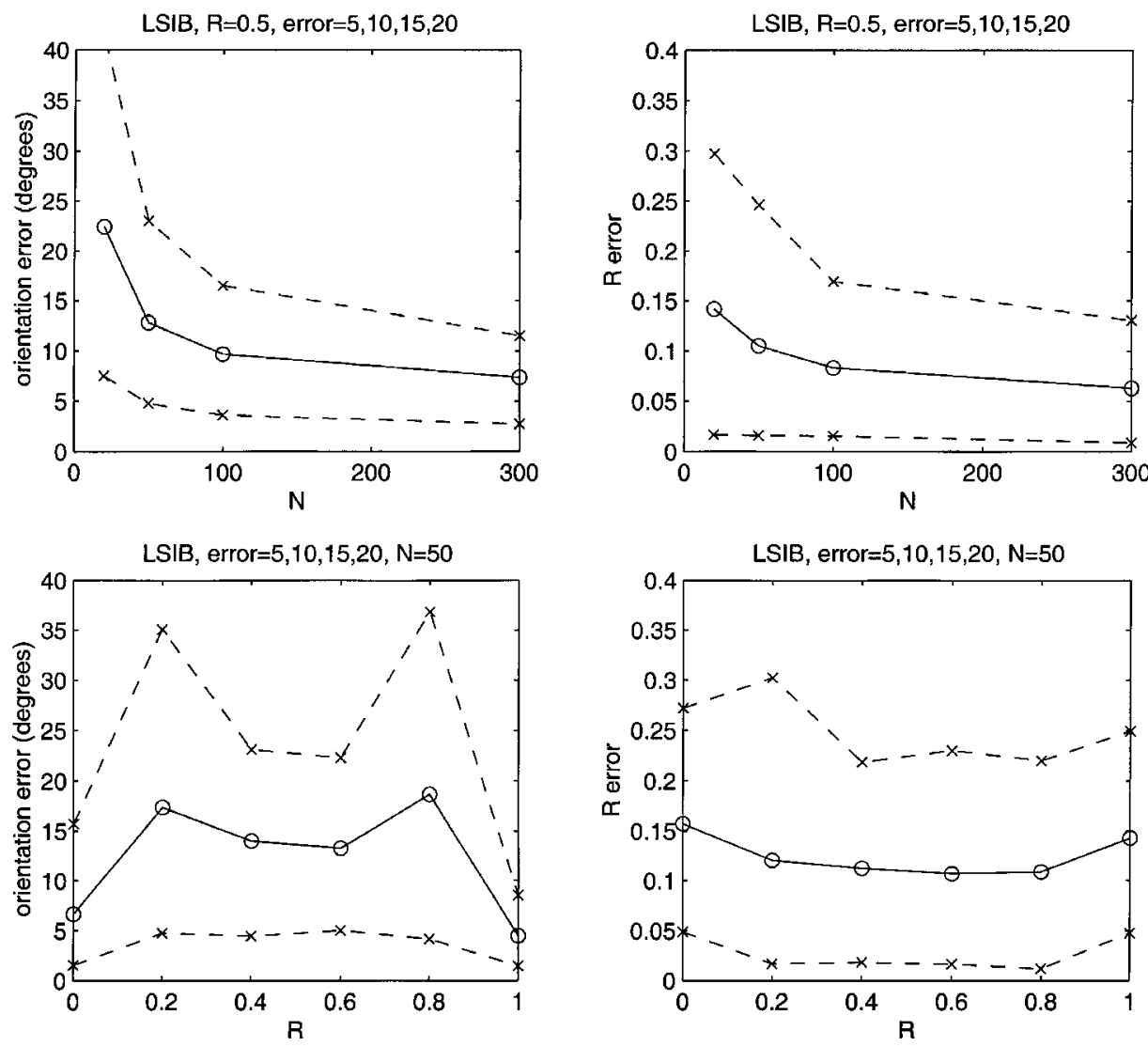

LSIB, $R=0.5, N=20,50,100,300$
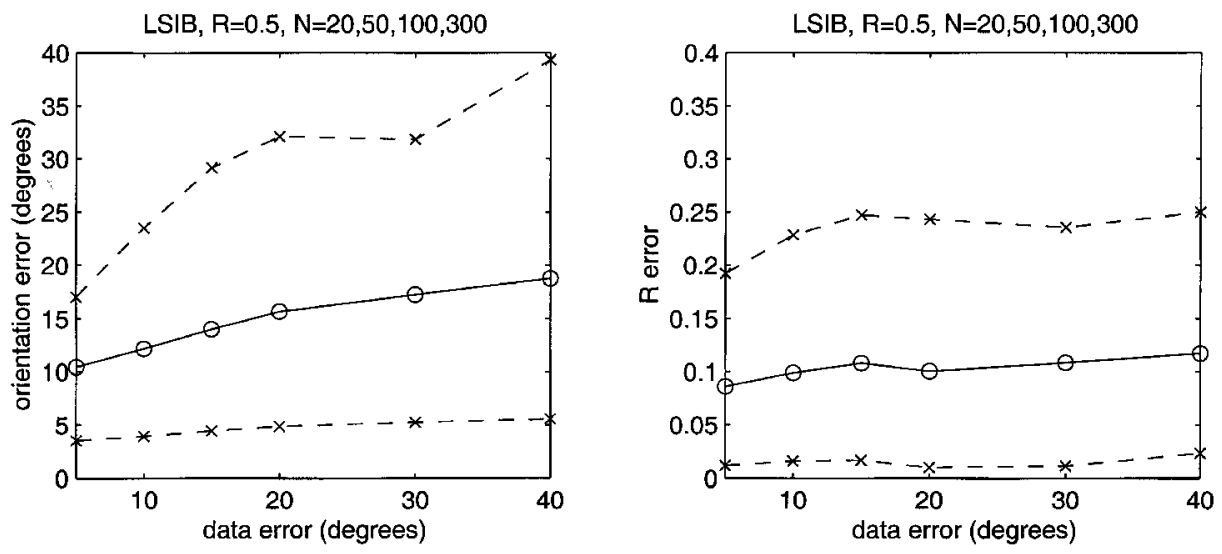

Figure 3. Accuracy of LSIB for synthetic data sets with the given values of $N$ (data set size), $R$, and mechanism error. Fifty synthetic data sets were created for each combination of parameter values listed. The synthetic data sets were created to be consistent with a given stress state according to the assumptions of the inversion methods, and random errors were added. The orientation error is defined as the angular misfit between the stress axes determined by inversion of the synthetic data and the stress axes used to create the data set. The $R$ error is the difference between the $R$ values of the two stress states. The circles connected by the solid line represent the mean error for the suite of data sets, and the crosses connected by the dashed lines encompass the middle $80 \%$. 

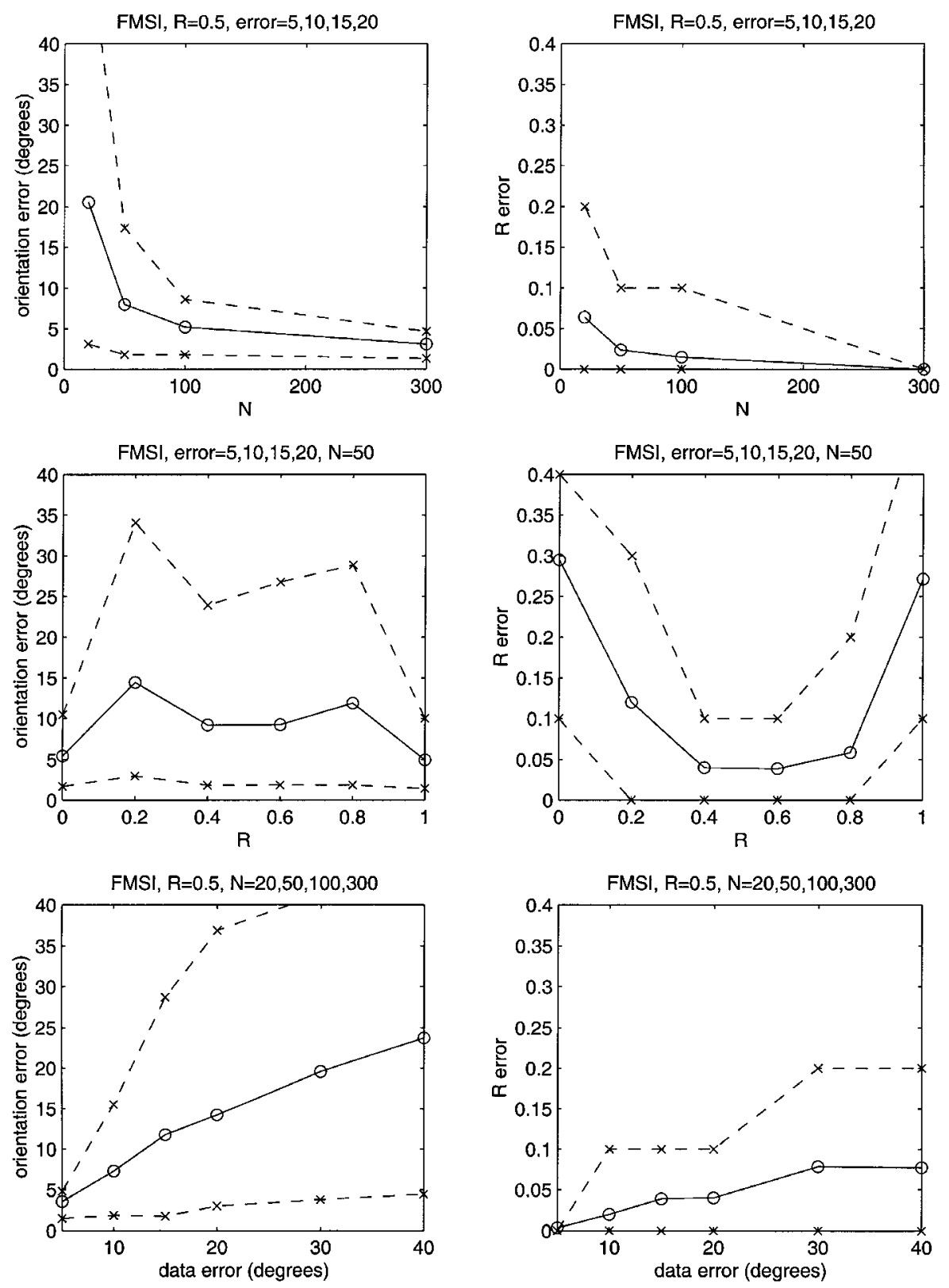

Figure 4. Accuracy of FMSI; symbols are as described in Figure 3.

considers the auxiliary planes only in error estimation, may also adversely affect the inversion accuracy if the data set contains a significant fraction of incorrectly picked planes. Surprisingly, the accuracy for very noisy data is quite good, with the average error less than that of FMSI and much less than the data error.

The LSIB confidence regions for large data sets $(N=$ 300 ) are too small. This may be because larger data sets often contain a number of similar focal mechanisms, which are essentially repeated data. These same data will also be repeated in the resampled data sets more often than other data. The full range of population samples, however, should repeat all data with similar frequency.
More representative resampled data sets can be made by sorting the mechanisms into bins based on strike, dip, and rake. Only one mechanisms from each nonempty bin is used in the base data set that is resampled during bootstrap error estimation. Although the base data sets contain $<300$ events, each resampled data set still contains 300 events. The confidence regions found using the modified resampling method are of more appropriate size (Fig. 9).

\section{Problems with FMSI}

The FMSI confidence regions are systematically too large. However, it is presently unclear why this is the case. Here we discuss the validity of the two major assumptions, 

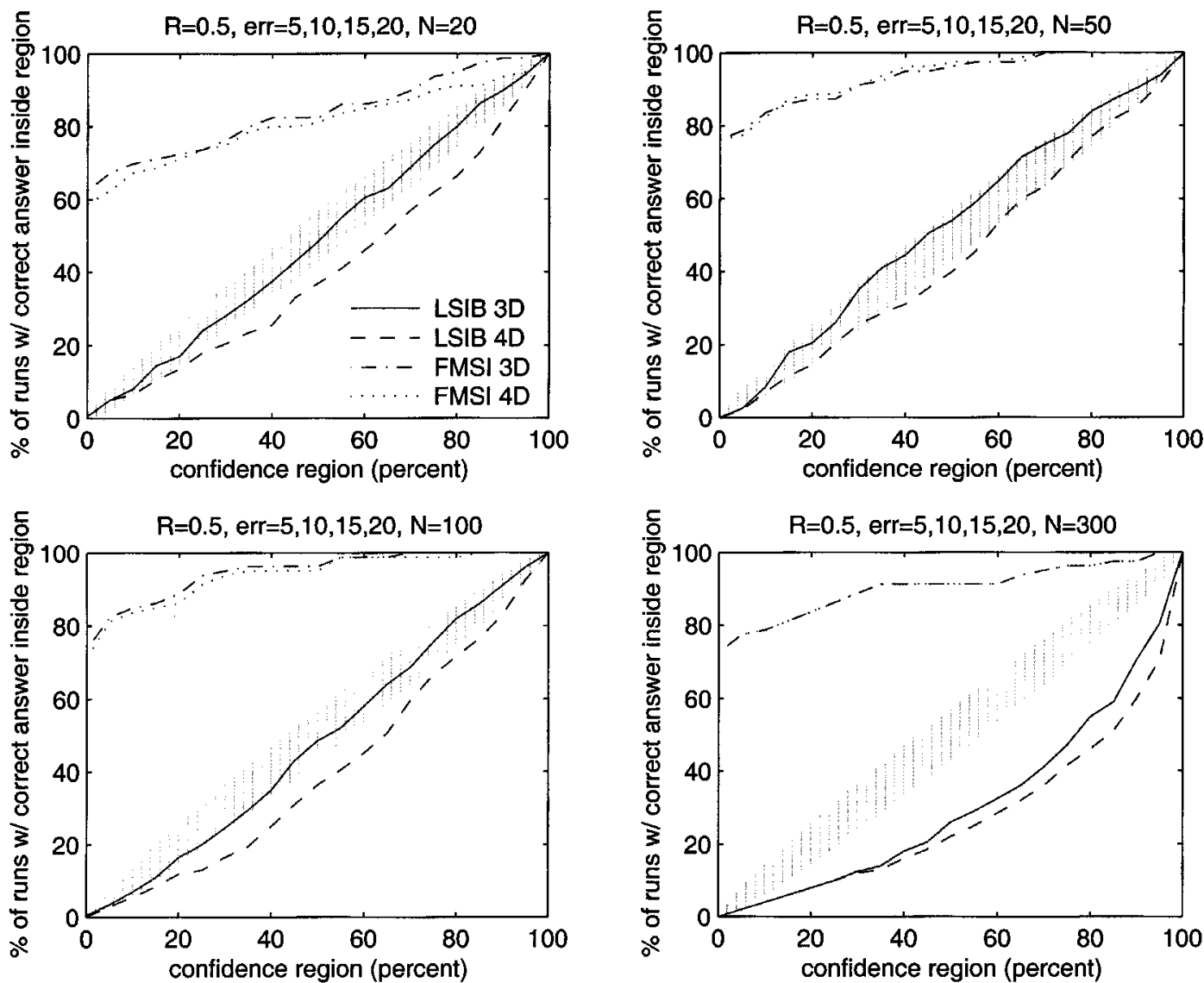

Figure 5. Confidence region appropriateness for both inversion methods, for varying $N$ (data set size.) The number of times the correct stress state falls within the $X \%$ confidence region is plotted as a function of $X$. If the confidence regions are appropriate, the function should fall within the shaded area. Results from an experiment with perfect confidence regions would have a $95 \%$ probability of falling within the shaded zone, which is the $95 \%$ confidence region of the binomial probability distribution for 200 trials with an $X \%$ probability of success each trial. If the confidence regions are too big or too small, the function will fall above or below the shaded area, respectively (see Fig. 2.) There are 50 data sets for each combination of parameter values listed. The orientations of the fault plane were chosen randomly. The label 4D indicates that all four model parameters were considered; 3D indicates only principal axis orientations.

normally distributed mechanism misfits due to data errors and a minimum observed misfit equal to the expected value of misfit for the best-fit stress state. We also consider the effects of finite grid spacing and nodal plane ambiguity. Finally, we discuss a method for recalibrating the uncertainties of FMSI in light of our numerical tests.

Error Distribution. It may be unrealistic to assume that the individual event misfits due to data errors are normally distributed whereas the data errors themselves are exponentially distributed. From the procedure followed in equations (6)-(11), we see that

$$
\frac{\Sigma_{X}}{\Sigma_{\min }} \approx \frac{z_{X} \sigma+E[m]}{E[m]}
$$

and that $\Sigma_{X}$ will scale with the ratio $\sigma / E[m]$. In the case of normally distributed misfits,

$$
\frac{\sigma}{E[m]}=\frac{(1-2 / \pi)^{1 / 2} N^{1 / 2}}{(2 / \pi)^{1 / 2} N} \approx 0.76 N^{-1 / 2}
$$

In the case of exponentially distributed misfits, we find numerically that

$$
\frac{\sigma}{E[m]} \approx \frac{(0.5 N)^{1 / 2}}{0.7 N} \approx N^{-1 / 2}
$$

Assuming an exponential distribution of misfits would therefore result in larger $\Sigma_{X}$ and larger confidence regions.

The distribution of event misfits for the correct stress 

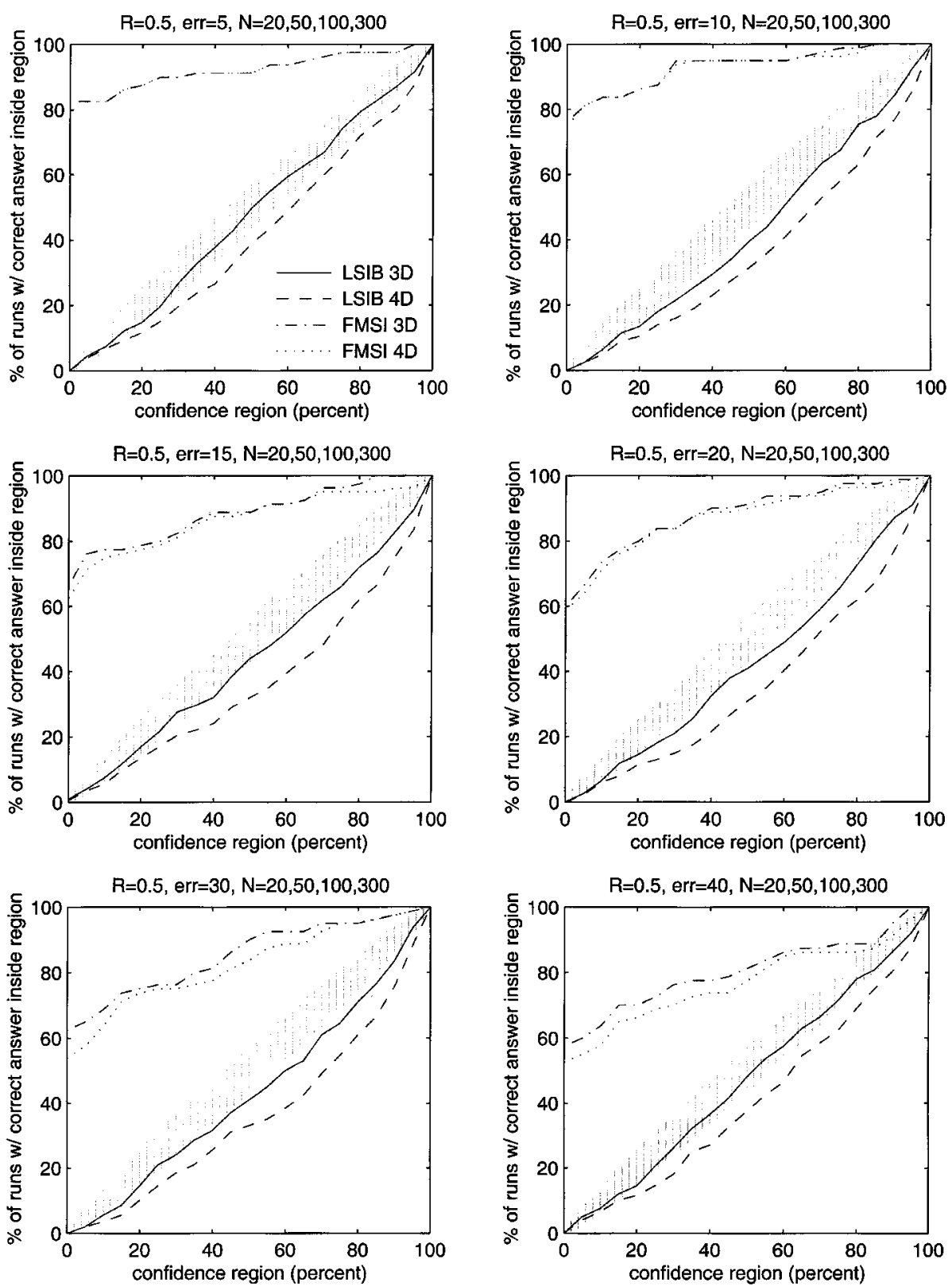

Figure 6. Confidence region appropriateness for varying focal mechanism error. Symbols are as described in Figure 5.

state need not be the same as the mechanism error distribution. For each event, the misfit for the correct stress state cannot be greater than the mechanism error. However, it can be less if the given mechanism is closer to another acceptable mechanism than to the correct mechanism for that event. We estimate the distribution of event misfits for the synthetic data sets by finding the minimum rotation angle between each mechanism and any focal mechanism compatible with the correct stress state. Choosing misfits from this distribution, we find numerically that

$$
\frac{\sigma}{E[m]} \approx \frac{(0.6 N)^{1 / 2}}{N} \approx 0.77 N^{-1 / 2} .
$$

Although the misfits are not normally distributed, the ratio $\sigma / E[m]$, which controls $\Sigma_{X}$ and the size of the confidence regions, is very similar to the ratio $\sigma / E[m]$ for a normal distribution. This implies that the assumption of a normal misfit distribution, while not strictly correct, is not the cause of the large confidence regions.

Expected Misfit. The computation of the threshold value of the confidence region, $\Sigma_{X}$, is based on the assumption that the minimum observed total misfit, $\Sigma_{\text {min }}$, is the expected value of the total misfit due to data errors for the correct stress state, $\sigma_{\text {est }} E[m]$. It is unrealistic to expect $\Sigma_{\min }$ to always exactly equal $\sigma_{\text {est }} E[m]$, but if $\Sigma_{\min }$ equals $\sigma_{\text {est }} E[m]$ on aver- 

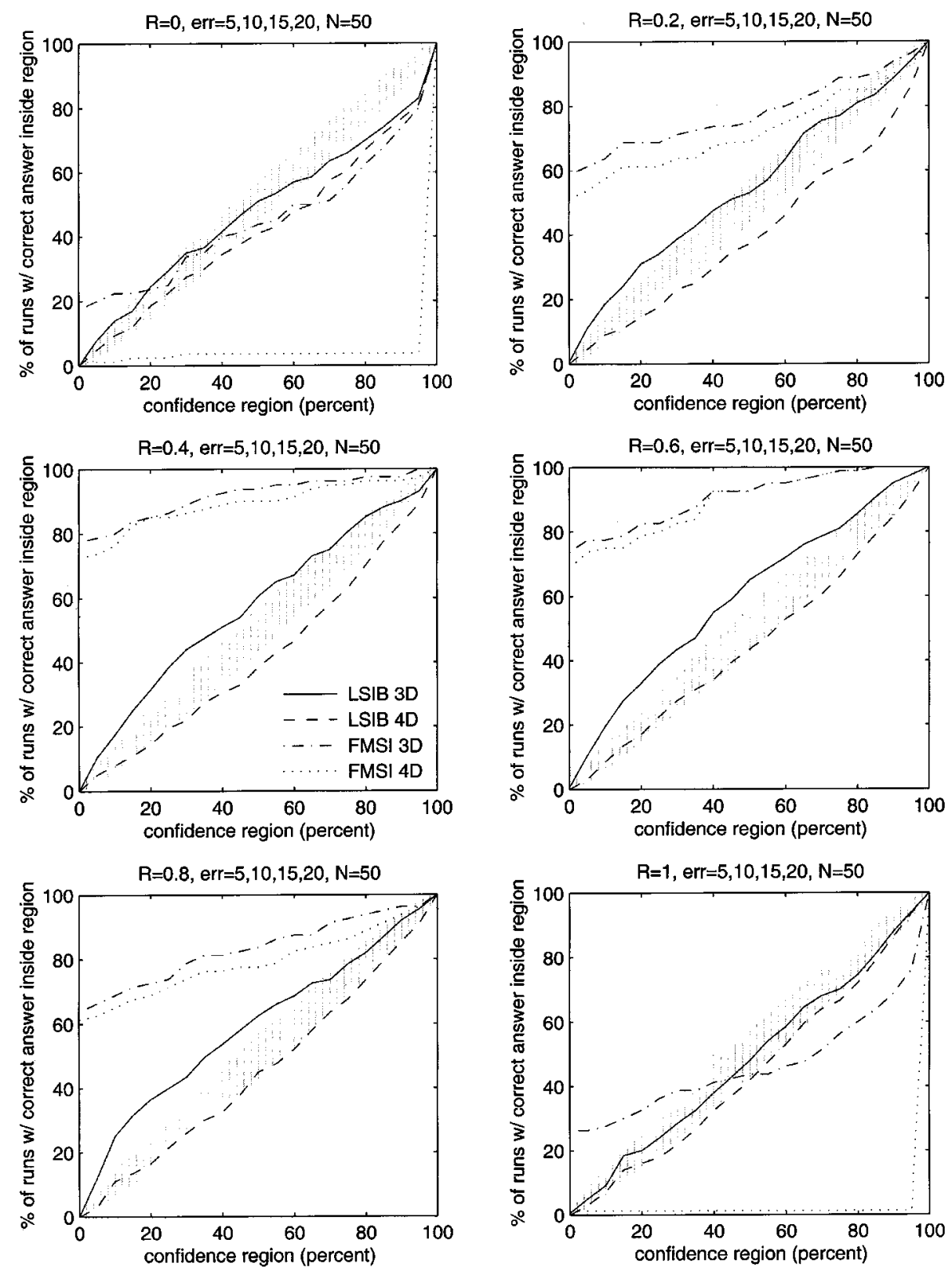

Figure 7. Confidence region appropriateness for varying $R$. Symbols are as described in Figure 5.

age, $\Sigma_{X}$ should be correct on average as well. To explain confidence regions that are systematically too large, $\Sigma_{\min }$ would have to be systematically greater than $\sigma_{\text {est }} E[m]$. This would imply that something besides focal mechanism error is contributing to the misfits. Usually, one would conclude that model errors are being mapped into the misfits. However, since the synthetic data sets were created to be consistent with the model assumptions, this should not be the case. It is unclear what an additional source of error could be.

Finite Grid Spacing. The finite gridding of parameter space can affect the apparent appropriateness of the uncertainty estimates in cases where the size of the grid spacing is larger than, or comparable to, the appropriate size of the $X \%$ confidence region. In this case, the X\% confidence region may appear too large even though it encompasses only one grid point. This is not a general explanation for the large confidence regions, however, because the 68\% and $95 \%$ confidence regions typically contain many grid points. Finite grid spacing can explain the large confidence regions only for cases of very high accuracy, $N=300$ or error $=5^{\circ}$, and low levels of confidence.

Fault Plane Ambiguity. Fault plane ambiguity may be responsible for the difficulty of FMSI in determining $R$ in the axisymmetric case ( $R=0$ or $R=1$.) Random slip orien- 

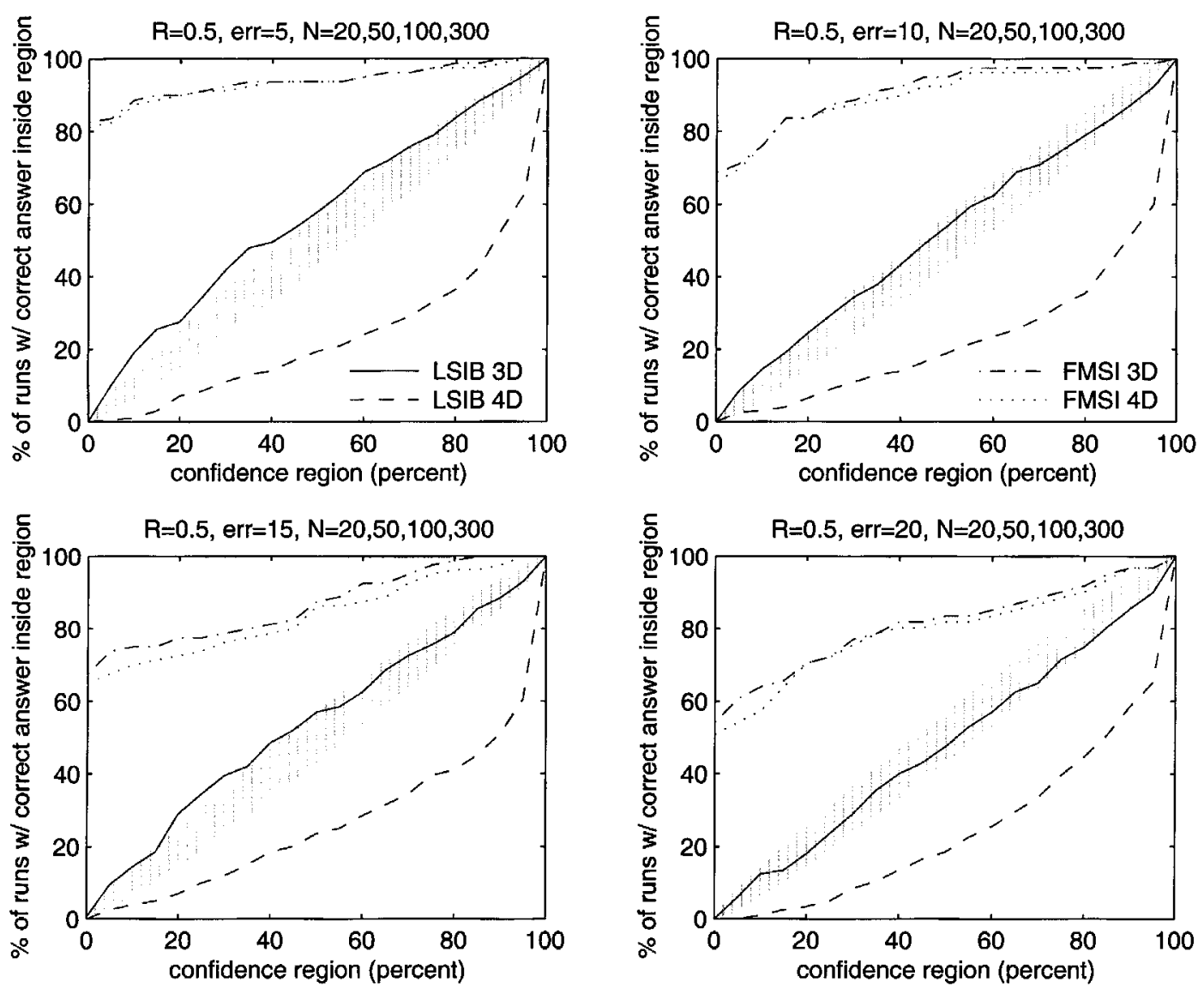

Figure 8. Confidence region appropriateness for varying focal mechanism error. The fault planes were chosen to be more uniform than in Figure 6, (see text). Symbols as in Figure 5.

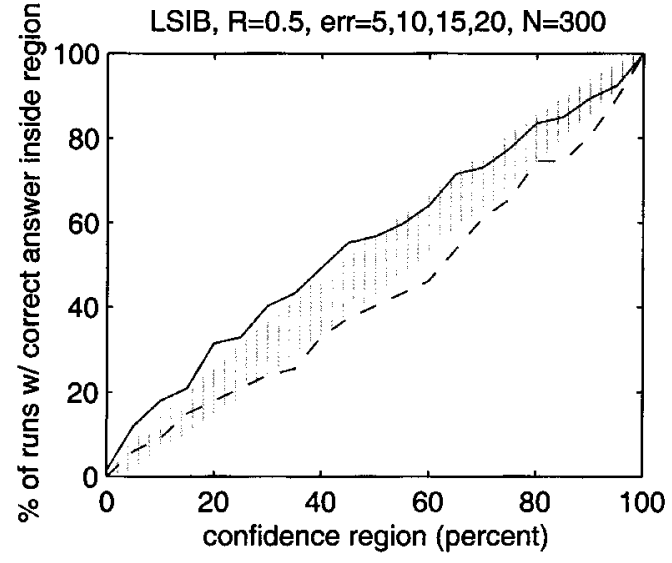

Figure 9. Confidence region appropriateness for LSIB for $N=300$ when a modified resampling method is used. The focal mechanisms are sorted into $10^{\circ}$-wide bins based on strike, dip, and rake. Only one mechanism from each nonempty bin is used in the base data set that is resampled during bootstrap error estimation. Although the base data set contains $<300$ events, each resampled data set still contains 300 events. Symbols are as described in Figure 5. tations tend to result in $R \approx 0.5$. The introduction of incorrect data, such as the auxiliary planes of some events, may move the computed value of $R$ toward the middle of its range. If only the correct fault planes are included in the data set, and the misfit is computed only for these planes, the accuracy of $R$ and the appropriateness of the uncertainty estimates improve (Figs. 10, 11). In general, however, modifying FMSI not to include auxiliary planes in the misfit computation does not improve its performance because the correct fault plane is usually not known.

Recalibration from Numerical Tests. Although it is currently unknown why the FMSI confidence regions are too large, numerical tests of the kind we performed can be used to recalibrate the uncertainty estimates. The results of the numerical tests provide a mapping between the FMSI confidence level $X$ ( $x$ axis) and the corresponding observed confidence level $Y$ ( $y$ axis). To use the misfit surface returned by FMSI to find the appropriate confidence region for confidence level $Y$, we apply this mapping in reverse.

We define the calibration factor, $c$, as the ratio between the corrected threshold value $\Sigma_{Y}^{\text {cor }}$ and the uncorrected value $\Sigma_{Y}^{\text {unc }}$, such that 
FMSI, $N=50, R=0$

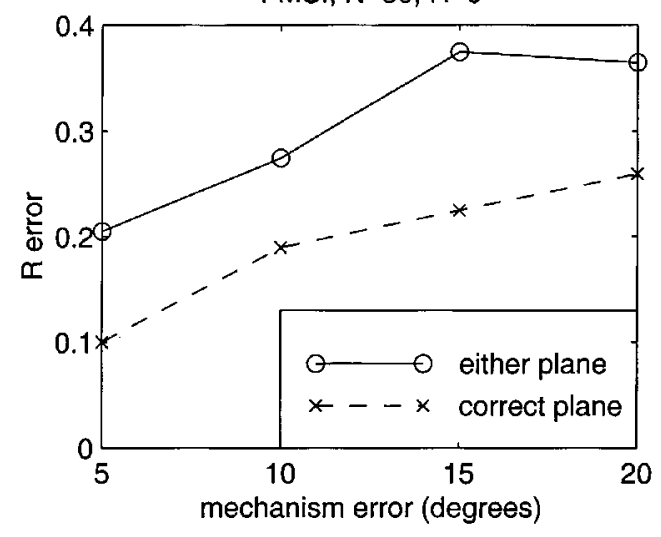

FMSI, $N=50, R=1$

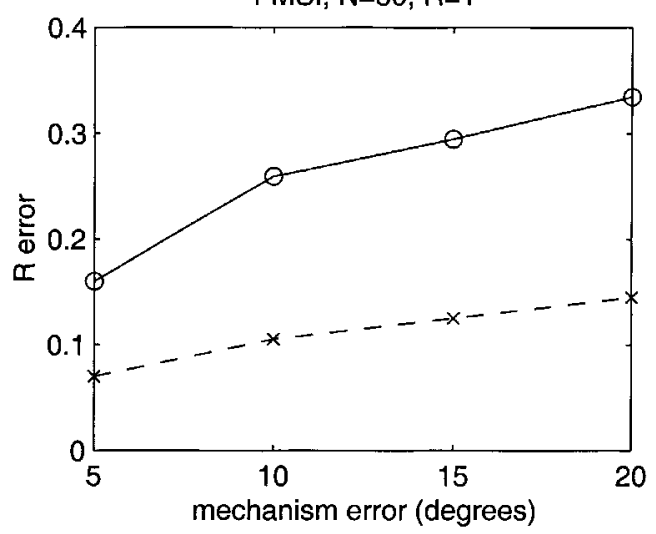

Figure 10. Average accuracy of $R$ determined by FMSI, for axisymmetric stress tensors $(R=0$ and $R=1)$ when the fault plane is ambiguous (circles and solid line) and known (crosses and dashed line). There are 50 data sets for each level of error.
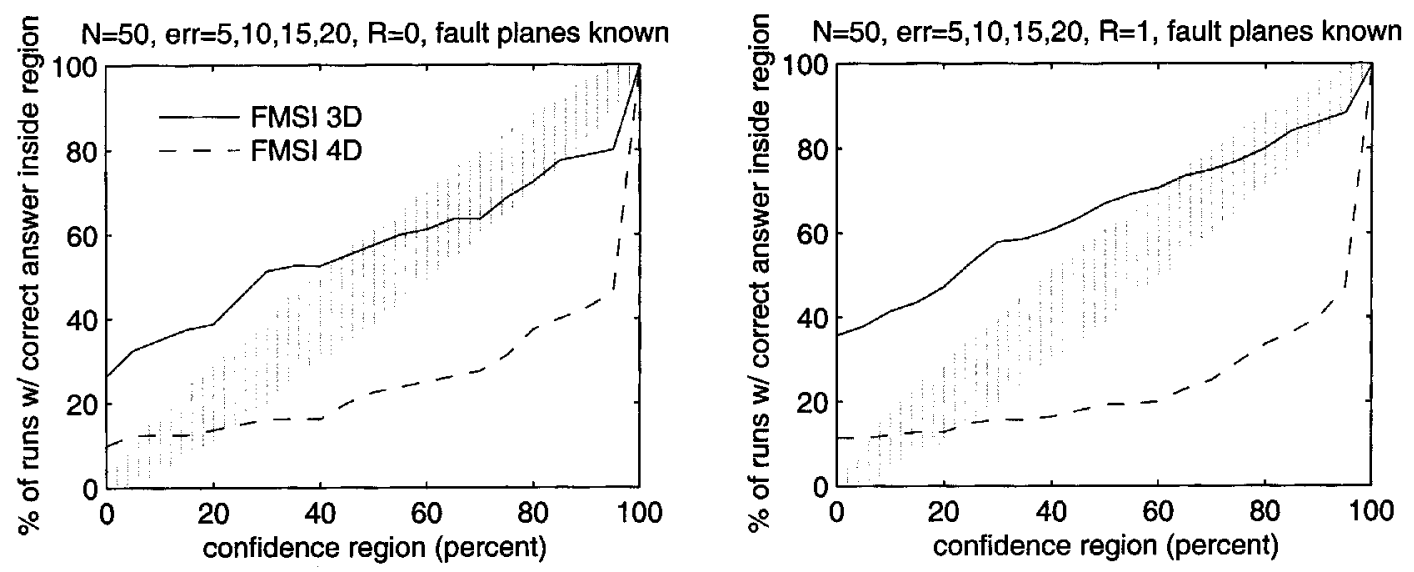

Figure 11. Confidence region appropriateness for FMSI for the axisymmetric case when the fault plane in known. Solid line, 3D; dashed line, 4D. All other symbols are as described in Figure 5. There are 50 data sets for each level of error.

$$
\Sigma_{Y}^{\mathrm{cor}}=c \Sigma_{Y}^{\mathrm{unc}}
$$

Using equation (11):

$$
\begin{aligned}
& \frac{\Sigma_{Y}^{\text {cor }}}{\Sigma_{\min }}=\frac{\Sigma_{X}}{\Sigma_{\min }}=\frac{z_{X}(\pi / 2-1)^{1 / 2} N^{1 / 2}+N}{N-4} \\
& =c\left(\frac{z_{Y}(\pi / 2-1)^{1 / 2} N^{1 / 2}+N}{N-4}\right)=c \frac{\Sigma_{Y}^{\mathrm{unc}}}{\Sigma_{\min }}
\end{aligned}
$$

so

$$
c=\frac{z_{X}(\pi / 2-1)^{1 / 2} N^{1 / 2}+N}{z_{Y}(\pi / 2-1)^{1 / 2} N^{1 / 2}+N} .
$$

The calibration factor, $c$, clearly depends on the data set size $N$, the $R$ value, the level of random error in the data, the confidence level of interest, and possibly other characteristics of the data that are not explored in this article. For our numerical tests with $R=0.5$ and error $=5^{\circ}, 10^{\circ}, 15^{\circ}$, and $20^{\circ}, c$ appears to strongly depend on $N$ and weakly depend on the other parameters. For $N=20, c \approx 0.929$; for $N=$ $50, c \approx 0.885$; for $N=100, c \approx 0.912$; and for $N=300$, $c \approx 0.959$. These estimated values of $c$ are very approximate and may not be appropriate for data sets that differ from the synthetic sets used to constrain them.

\section{Conclusions}

The two most commonly used techniques for inverting earthquake focal mechanisms for stress orientation, FMSI (Gephart and Forsyth, 1984; Gephart, 1990a) and LSIB (Michael, 1984, 1987b), were tested on noisy synthetic data sets. Both techniques determine stress orientation accurately. The FMSI method is generally more accurate for high-quality data, whereas LSIB is more accurate for very noisy data. The confidence regions produced by LSIB are usually approxi- 
mately the right size, whereas those of FMSI are usually too large.

The results for the synthetic data sets indicate that the confidence regions produced by LSIB should be appropriate for real data sets as well, as long as the model assumptions of homogeneous stress and slip in the direction of resolved shear stress generally hold. The numerous stress field variations that have been observed in southern California using LSIB (Michael, 1987b; Jones, 1988; Hauksson, 1990, 1994; Kerkela and Stock, 1996; Hardebeck and Hauksson, 1999, 2001) are therefore larger than the inversion uncertainty and probably represent real signals. The crustal stress field in a region can vary in space and time, and earthquake focal mechanisms can reliably be used to detect these variations.

\section{Acknowledgments}

We thank Andrew Michael and John Gephart for valuable discussions and for providing their inversion codes, and Max Wyss and an anonymous reviewer for their helpful comments on the manuscript. This research was supported by the Southern California Earthquake Center (SCEC). SCEC is funded by NSF Cooperative Agreement EAR-8920136 and USGS Cooperative Agreements 14-08-0001-A0899 and 1434-HQ-97AG01718. The SCEC Contribution Number for this paper is 504. Contribution Number 8706, Caltech Division of Geological and Planetary Sciences.

\section{References}

Abers, G. A., and J. W. Gephart (1997). Stress variations in southern California determined by direct inversion of seismic first motions (MOTSI), EOS Trans. AGU 78, F451.

Gephart, J. W. (1990a). FMSI: A FORTRAN program for inverting fault/ slikenside and earthquake focal mechanism data to obtain the regional stress tensor, Computers and Geosciences 16, 953-989.

Gephart, J. W. (1990b). Stress and the direction of slip on fault planes, Tectonics 9, 845-858.

Gephart, J. W., and D. W. Forsyth (1984). An improved method for determining the regional stress tensor using earthquake focal mechanism data: application to the San Fernando earthquake sequence, J. Geophys. Res. 89, 9305-9320.

Gillard, D., M. Wyss, and P. Okubo (1996). Stress and strain tensor orientations in the south flank of Kilauea, Hawaii, estimated from fault plane solutions, J. Geophys. Res. 100, 16,025-16,042.

Hardebeck, J. L., and E. Hauksson (1999). Role of fluids in faulting inferred from stress field signatures, Science $\mathbf{2 8 5}$, 236-239.

Hardebeck, J. L., and E. Hauksson (2001). The crustal stress field in southern California and its implications for fault mechanics, J. Geophys. Res. (submitted).
Hauksson, E. (1990). Earthquakes, faulting, and stress in the Los Angeles basin, J. Geophys. Res. 95, 15,365-15,394.

Hauksson, E. (1994). State of stress from focal mechanisms before and after the 1992 Landers earthquake sequence, Bull. Seism. Soc. Am. 84, 917-934.

Jones, L. M. (1998). Focal mechanisms and the state of stress on the San Andreas Fault in Southern California, J. Geophys. Res. 93, 88698891.

Kerkela, S., and J. M. Stock (1996). Compression directions north of the San Fernando Valley determined from borehole breakouts, Geophys. Res. Lett. 23, 3365-3368.

Lu, Z., M. Wyss, and H. Pulpan (1997). Details of stress directions in the Alaska subduction zone from fault plane solutions, J. Geophys. Res. 102, 5385-5402.

Lund, B., and R. Slunga (1999). Stress tensor inversion using detailed microearthquake information and stability constraints: application to Olfus in southwest Iceland, J. Geophys. Res. 104, 14,947-14,964.

Michael, A. J. (1984). Determination of stress from slip data: faults and folds, J. Geophys. Res. 89, 11,517-11,526.

Michael, A. J. (1987a). Use of focal mechanisms to determine stress: a control study, J. Geophys. Res. 92, 357-368.

Michael, A. J. (1987b). Stress rotation during the Coalinga aftershock sequence, J. Geophys. Res. 92, 7963-7979.

Michael, A. J. (1991). Spatial variations in stress within the 1987 Whittier Narrows, California, aftershock sequence: new techniques and results, J. Geophys. Res. 96, 6303-6319.

Michael, A. J. (2000). A genetic algorithm stress inversion method, Seism. Res. Lett. 71, 228.

Parker, R. L., and M. K. McNutt (1980). Statistics for the one-norm misfit measure, J. Geophys. Res. 85, 4429-4430.

Pollard, D. D., S. D. Saltzer, and A. M. Rubin (1993). Stress inversion methods: are they based on faulty assumptions? J. Structural Geol. 15, 1045-1054.

Reasenberg, P., and D. Oppenheimer (1985). FPFIT, FPPLOT and FPPAGE: FORTRAN computer programs for calculating and displaying earthquake fault-plane solutions, U. S. Geol. Surv. Open-File Rept. 85-730, 109.

Wyss, M., and Z. Lu (1995). Plate boundary segmentation by stress directions: southern San Andreas fault, California, Geophys. Res. Lett. 22, 547-550.

Wyss, M., B. Liang, W. R. Tanigawa, and X. Wu (1992). Comparison of orientations of stress and strain tensors based on fault plane solutions in Kaoiki, Hawaii, J. Geophys. Res. 97, 4769-4790.

Zhao, D., H. Kanamori, and D. Wiens (1997). State of stress before and after the 1994 Northridge earthquake, Geophys. Res. Lett. 24, 519522 .

Seismological Laboratory MC 252-21

California Institute of Technology

Pasadena, California, 91125

Manuscript received 9 March 2000. 